\title{
Self-motion in glass-forming polymers: A molecular dynamics study
}

\author{
A. van Zon and S. W. de Leeuw \\ Department of Applied Physics, Delft University of Technology, Lorentzweg 1, 2628 CJ Delft, The Netherlands
}

(Received 26 February 1999)

\begin{abstract}
We present results of molecular dynamics simulations of an undercooled polymer melt, performed to study the validity of mode-coupling theory (MCT) for realistic polymer melts in general. The mean square displacements of the chain segments are computed to study the diffusion constant of the Rouse-like motion. It is shown that this diffusion constant follows a power law behavior as a function of the temperature, as predicted by the MCT. In addition, we studied the incoherent part of the intermediate scattering function and show that these functions obey the second scaling law of the MCT. We also calculated the relaxation times of the $\alpha$-relaxation and found that they follow the same power law $(\gamma=2.9)$ as the diffusion constant. Using $\gamma$, and the relationships given by MCT, we obtain values for $a(0.27)$ and $b(0.46)$ and use these exponents to describe the $\beta$-relaxation regime. We find that the long time part of the $\beta$-relaxation can be described accurately by the Von Schweidler relaxation over a wide range of wave numbers. In the short time regime of the $\beta$-relaxation, no critical decay is observed. [S1063-651X(99)08111-8]
\end{abstract}

PACS number(s): 64.70.Pf, 61.25.Hq, 61.20.Ja

\section{INTRODUCTION}

Over the past decade or so, much progress has been made in understanding the dynamics of liquids in the undercooled regime. This started with the work of Leutheusser [1] and Bengtzelius et al. [2], who used a self-consistent kinetic theory to show that dynamical quantities of liquids change rapidly near a critical temperature, which can be interpreted as the glass transition temperature. This mode-coupling theory (MCT) was extended in later years [3] and lead to a variety of experimental and numerical work.

Using molecular dynamics (MD), which has been a useful tool to understand the dynamics of dense liquids, many of the predictions of MCT have been tested in cases of simple monoatomic liquids $[4,5]$, binary liquid mixtures $[6,7]$, diatomic molecules [8], and water [9]. These simulations show that MCT provides a suitable theoretical framework for analyzing the dynamical properties in the undercooled regime, and that it is not limited to monoatomic liquids.

Experimentally, the relaxation of glass forming liquids can be studied using inelastic neutron scattering techniques and light scattering techniques. Because of the low crystallization tendency, many of these experiments are performed in polymer systems [10]. Although these experiments are in qualitative agreement with the most general predictions of the MCT, a dramatic slowing down of the dynamics near the glass transition, it is difficult to obtain more detailed quantitative information. In addition, experiments show deviations from MCT. Probably the most important is the existence of a secondary relaxation, the Johari-Goldstein process, which is believed to be caused by intramolecular movement [11].

In the case of polymer melts the dynamics outside the undercooled regime differs from that of simple liquids [12]. It is well known that in the case of unentangled polymers, the dynamics shows a Rouse-like behavior caused by structural relaxations of the chain, i.e., motion of sites in the center of mass of the polymer. When the length of the polymers is increased, the dynamics changes to a more complicated reptationlike motion [13]. It is believed that this is caused by the formation of a tube which suppresses the lateral movement of the polymer. MD studies of polymers are usually performed in the framework of this Rouse or reptation dynamics, or when the glass transition is studied, in a phenomenological way [14]. Only recently, analysis of glass forming polymers in the framework of MCT have been performed $[15,16]$. These studies show, in addition to experimental results and Monte Carlo calculations [17], that MCT can be applied to the dynamics of undercooled polymer melts. Recently, Bennemann et al. performed a detailed study of an undercooled polymer melt [18]. They found that the intermediate scattering function could be described accurately within the framework of MCT. However, due to the use of a simple bead-spring model, there are some differences with experimental results. In this study, a realistic model is used to describe the polymer dynamics. From direct comparison with neutron scattering experiments it is well known that these kinds of realistic models are able to describe the intermediate scattering function $[15,19,20]$, which is the property of interest in this article. Usually, these simulations are performed at high temperatures, well above the glass transition temperature.

In this article we show that the dynamics of a realistic undercooled polymer melt can be understood in great detail within the framework of MCT. To perform a detailed test we focus on the relationship between the $\alpha$-relaxation and the $\beta$-relaxation regime. According to MCT, both regimes are characterized by a single parameter, the exponent parameter $\lambda$ (see Sec. II for more details). The other parameters are related to $\lambda$. Therefore, obtaining $\lambda$ from the $\alpha$-relaxation regime, one can check the predictions of MCT in the $\beta$-relaxation regime. In this article we focus on the selfmotion of the chain segments. Collective properties will be analyzed in a future publication. In addition, our results are compared to experiments on glass-forming polymers. We show that the use of a more realistic interaction potential leads to a good agreement with experimental results, and therefore to a better understanding of the dynamics of glassforming polymers. The discrepancy between experimental 
results and MD simulations, as found in Ref. [18], can be explained in terms of polymer size and interaction.

The outline of this paper is as follows. In Sec. II we formulate the results of MCT. Then, in Sec. III the model and the simulation details will be discussed. In Sec. IV we give our results, and finally in Sec. V we discuss these results.

\section{MODE-COUPLING THEORY}

In this section we briefly summarize the main ideas and the results of the idealized MCT. A complete overview of this theory can be found in Ref. [3].

The property of interest is the intermediate scattering function, $F(k, t)$, which is the Fourier transform of the Van Hove correlation function $G(r, t)$. Since the Van Hove correlation function consists of two parts, a self part $G^{s}(r, t)$, which described the diffusive motion of the particles, and a collective part $G^{d}(r, t)$, which describes the collective motion, its Fourier transform also contains two parts. In this article we focus on the self part of $F(k, t)$,

$$
F^{s}(k, t)=\int d \mathbf{r} G^{s}(r, t) e^{i \mathbf{k} \cdot \mathbf{r}}=\left\langle e^{-i \mathbf{k} \cdot\left[\mathbf{r}_{i}(t)-\mathbf{r}_{i}(0)\right]}\right\rangle,
$$

where $\langle\ldots\rangle$ denotes standard ensemble averaging. In the case of polymer dynamics the self part of $F(k, t)$ describes the diffusive motion of the chain segments.

The starting point of the MCT is the generalized Langevin equation (GLE), obtained by using the projection operator formalism of Mori and Zwanzig [21]. This GLE, which is still exact, describes the decay of dynamical variables and correlation functions, in terms of a memory function $M(k, t)$. Unfortunately, these equations are not exactly solvable and approximations have to be made. Within the mode-mode coupling approximation, the memory function of the GLE is written in terms of products of the correlation functions itself. This idea is supposed to work best for (a set of) slowly varying dynamical variables; the hydrodynamic variables. Products of correlation functions in the memory function can describe different physical relaxation mechanisms. A well known example is the product of transverse current correlation functions and self intermediate scattering functions. This product describes the backflow effect and leads to the well known long time tails [22]. The cage-effect, which becomes very important in undercooled liquids, can be described with products of the intermediate scattering function. In the idealized version of MCT, only these products are used to describe the memory function. This leads to a closed set of coupled integro-differential equations with nonlinear feedback. When $k$-dependence is neglected, only one equation is left which can be analyzed with relative ease analytically as well as numerically $[1,2]$. It has been shown that this nonlinear feedback can lead to a transition from ergodic to nonergodic behavior for certain coupling strengths of the nonlinear feedback. The latter depends on both temperature and number density. In the following we take the number density constant (simulations are performed at constant volume). As a result this transition occurs at a certain temperature $T_{c}$, which is called the critical temperature.
When $k$-dependence is not neglected, one has to deal with a set of coupled nonlinear differential equations for which analytical results can no longer be obtained. However, numerical studies show that the basic idea, a crossover from ergodic to nonergodic behavior, is still the same. An important step in analyzing this crossover is the reduction formalism $[3,23]$. As a result, close to the critical temperature and in the $\beta$-relaxation regime, $F(k, t)$ can be described by one single relaxation function $G(t)$, which is related to $F(k, t)$ via

$$
F^{s}(k, t)=f_{k}+h_{k} G(t),
$$

where $f_{k}$ is the nonergodicity parameter and $G(t)$ is usually referred to as the $\beta$-correlator. Close to the glass transition, $G(t)$ obeys the so-called first scaling law,

$$
G(t)=c_{\epsilon} g\left(t / t_{\epsilon}\right)
$$

where $\epsilon$ is the separation parameter $\epsilon=\left(T-T_{c}\right) / T_{c}$, which gives the relative separation from the critical temperature. $t_{\epsilon}$ is the time scale at which the $\beta$-relaxation occurs. On the liquid side of the glass transition, an equation can be derived for $g\left(t / t_{\epsilon}\right)$ in the above defined scaling region [3],

$$
\frac{1}{\tilde{z}}+\frac{1}{\lambda} \tilde{z} g^{2}(\tilde{z})+\mathcal{L}\left\{g^{2}(\tilde{t})\right\}=0,
$$

where $\tilde{t}=t / t_{\epsilon}, \tilde{z}=z / \omega_{\epsilon}$, and $\mathcal{L}$ denotes the Laplace transform. $g\left(t / t_{\epsilon}\right)$ now only depends on $\lambda$, the exponent parameter. In principle, $\lambda$ can be calculated from the static structure factor, but here we shall use it as a free parameter. The usual way to analyze Eq. (4) is to expand $g\left(t / t_{\epsilon}\right)$ for $t \ll t_{\epsilon}$ like

$$
g\left(t / t_{\epsilon}\right)=\left(t / t_{\epsilon}\right)^{-a}-A_{1}\left(t / t_{\epsilon}\right)^{a},
$$

and for $t \gg t_{\epsilon}$ like

$$
g\left(t / t_{\epsilon}\right)=-B\left(t / t_{\epsilon}\right)^{b}+B_{1}\left(t / t_{\epsilon}\right)^{-b},
$$

where $a \in[0,0.5]$ and $b \in[0,1]$. By substituting these expansions in Eq. (4) and sorting out equal powers of $t / t_{\epsilon}$, one finds that the parameters $a, b$, and $\lambda$ are related via

$$
\lambda=\frac{\Gamma(1-a)^{2}}{\Gamma(1-2 a)}=\frac{\Gamma(1+b)^{2}}{\Gamma(1+2 b)},
$$

where $\Gamma(x)$ denotes the usual $\Gamma$-function. Finally, $c_{\epsilon}$ and $t_{\epsilon}$ depend on $\epsilon$ like

$$
\begin{gathered}
c_{\epsilon}=\sqrt{|\epsilon|}, \\
t_{\epsilon}=t_{0}|\epsilon|^{-1 / 2 a} .
\end{gathered}
$$

Here $t_{0}$ is a free parameter that depends on the short time dynamics, binary collisions, and is, in principle, temperature dependent. On the $\alpha$-relaxation timescale the relaxation function can be described by a master function of the type

$$
F(k, t, T)=\widetilde{F}(k, t / \tau(T)) .
$$


From experiments [24] and simulations $[6,8,9,15,16]$ it is known that this master function can be described accurately by a stretched exponent,

$$
\widetilde{F}(k, t / \tau(T))=c_{k} e^{-(t / \tau)^{\beta}} .
$$

Also from numerical evaluation [25] it is known that this stretched exponential function gives a good description of the $\alpha$-relaxation. The temperature dependence of the relaxation time $\tau$ is the same as the one for the long time part of the $\beta$-relaxation and is determined by the exponents $a$ and $b$ according to

$$
\tau=|\epsilon|^{\gamma / t_{0}},
$$

where

$$
\gamma=1 / 2 a+1 / 2 b .
$$

We now have a complete description of the dynamics close to the critical temperature, which holds for both the coherent and the incoherent part of $F(k, t)$. It should be noted that no specific aspects of polymer dynamics (i.e., Rouse dynamics) are included in this theory. However, the theory includes the static structure factor which contains information about the structural properties of the polymer melt. But this structure factor only influences the numerical values of the parameters.

\section{MODEL AND SIMULATION}

In our simulations we use a system containing 24 linear polymer chains of 100 sites each. Each of these sites represents a group in the chain, for example a $\mathrm{CH}_{2}$-group, so hydrogen atoms are not taken into account explicitly. No distinction is made between "bulk" sites and "end" sites. The distances between two connected sites is $1.53 \AA$ and is fixed during the simulation. The mass of the atoms is 14 $(12+2)$ a.u. To introduce a certain amount of chain stiffness, we make use of valence angle and torsion angle potentials, of which the general form is shown in Eqs. (14) and (15). For the nonbonded interactions a Lennard-Jones potential is used [Eq. (16)]

$$
\begin{gathered}
V_{\mathrm{v} a l}(\theta)=\frac{k_{\theta}}{2}\left(\cos \theta-\cos \theta_{0}\right)^{2}, \\
V_{\text {tors }}(\phi)=\sum_{i=0}^{3} a_{i} \cos ^{n} \phi, \\
V_{L J}(r)=4 \epsilon\left[\left(\frac{\sigma}{r}\right)^{12}-\left(\frac{\sigma}{r}\right)^{6}\right] .
\end{gathered}
$$

Here $\theta$ is the valence angle, $\phi$ the torsion angle, and $r$ the distance between two nonbonded sites. $k_{\theta}$ in the valence angle potential is related to the small angle force constant $k$ via $k_{\theta}=k / \sin ^{2} \theta_{0}$. In our simulations we used $k_{\theta}$ $=133 \mathrm{kcal} / \mathrm{mol}$, which corresponds to $k=100 \mathrm{kcal} / \mathrm{mol}$. In this way the potential barrier for rotation about valence angles is $16.67 \mathrm{kcal} / \mathrm{mol} . \theta_{0}$ is set at $120^{\circ}$. The torsion angle potential contains three minima, one corresponding to a cis configuration and two corresponding to gauche configura- tions. The potential barrier between these minima is set at 2 $\mathrm{kcal} / \mathrm{mol}$. This leads to the following numerical prefactors: $a_{0}=1, a_{1}=-3, a_{2}=0$, and $a_{3}=4 \mathrm{kcal} / \mathrm{mol}$. The $\sigma$ of the Lennard-Jones potential is set at $4 \AA$ and $\epsilon$ at $0.25 \mathrm{kcal} / \mathrm{mol}$. This model is close to the one used by Roe in simulations of poly(ethylene) [26], but differs from the model used by Bennemann et al. [16], who neglected the valence and torsion potentials in their model. Both Roe and Bennemann et al. use springs to connect two neighboring sites in the chain.

The starting configuration is made using a pivot-Monte Carlo algorithm with a Metropolis acceptance criterion [27]. In this way the 24 chains of 100 carbon atoms are made. 100000 pivot moves per chain are performed in order to avoid correlation between the chains. After this the 24 polymers are brought together in a computational box (cube) with a size of $39.3 \AA^{3}$, corresponding to a density of $0.92 \mathrm{~g} / \mathrm{mol}$. Excluded volume is gradually "switched on" using a truncated Lennard-Jones potential. Finally the system is equilibrated for $4.4 \mathrm{~ns}$ using molecular dynamics at $T=0.7$, which corresponds to $528 \mathrm{~K}$. In these simulations the bond-lengths between the carbon atoms are kept fixed using a constraint dynamics algorithm [28]. The simulations are performed in a NVT ensemble in which the temperature is regulated via a Nosé-Hoover thermostat [29].

To get an indication of the glass transition temperature $T_{g}$, the total energy of the system is determined during a fast quench. In this quench, the system is cooled in steps of 0.01 , corresponding to $7.5 \mathrm{~K}$, and at each temperature the total energy is measured during $44 \mathrm{ps}$. At the glass transition temperature, the slope of the energy as a function of the temperature changes, due to a change in specific heat. In this way we find $T_{g}=0.255 \pm 0.01$, which corresponds to $T_{g}$ $=192 \mathrm{~K}$.

After equilibration, the system is cooled stepwise from $T=0.7$ to $T=0.330$ via temperatures $0.6,0.525,0.469$, $0.427,0.395,0.371,0.353$, and 0.340 . At each temperature the system is equilibrated for $2.2 \mathrm{~ns}$ and measurements are performed for $4.4 \mathrm{~ns}$. It is important to ensure that our system is in equilibrium at all temperatures. To check this condition the system is equilibrated at $T=0.330$, which is the lowest temperature in our calculations, for another $11 \mathrm{~ns}$. Then $F^{s}(k, t)$ is again measured for $400 \mathrm{ps}$ and compared to the $F^{s}(k, t)$ from the original configuration. This is shown in Fig. 1, where $F^{s}(k, t)$ is shown for three different $k$ values, before and after the additional equilibration. It is clearly visible that an additional equilibration of $11 \mathrm{~ns}$ has no significant effect on $F^{s}(k, t)$. This means that our system is at, or at least very close, to equilibrium.

\section{RESULTS}

\section{A. Structural properties}

To get information about the structural properties of the system we calculated the radial distribution function and the static structure factor. The static structure factor $S(k)$, which is shown in Fig. 2 for $T=0.7$, is similar to that of monatomic liquids until about $k=4 \AA^{-1}$. The first peak is situated at approximately $1.5 \AA^{-1}$ or, with $\sigma=4 \AA$, at $k \sigma=6$. The second peak is situated at approximately $k=3 \AA^{-1}$, twice the $k$ value of the first peak. After this second peak $S(k)$ is different from that of simple liquids because of the existence 


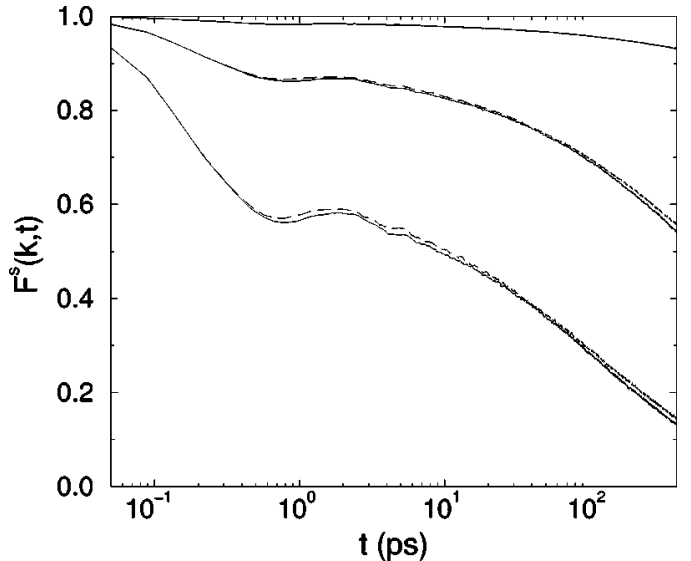

FIG. $1 . F(k, t)$ at $T=0.330$ for $k=2.88,1.44$, and $0.48 \AA^{-1}$ (left to right). The solid and dashed lines correspond to $F(k, t)$ before and after the additional equilibration for $11 \mathrm{~ns}$, respectively.

of relatively large oscillations. Only the first of these oscillations is shown in Fig. 2. The origin of these oscillations can be explained via the radial distribution function. The radial distribution function $g(r)$, shown in the inset in Fig. 2 for $T=0.7$ and $T=0.330$, is more complicated. At small distances, intramolecular correlations are visible. (The $\delta$-function peak at the bond length is removed from the plot.) For example, the peak at $r=2.6 \AA$ is caused by next nearest neighbors in the polymer chain. This distance is fixed because of the high energy barrier $(16.67 \mathrm{kcal} / \mathrm{mol})$ between the two energy minima in the valence angle. The absence of a high peak around $r=\sigma$ shows that the local packing, which is a common feature in dense liquids, is hindered by chain connectivity. Apart from the small bumps caused by atoms further away in the polymer, $g(r)$ can be split into three parts: One part originating from intermolecular correlations, a sharp peak at $r=2.6 \AA$ and a $\delta$-function peak at $r$ $=1.53 \AA$. By using the relation between $g(r)$ and $S(k)$ it can be shown that the $\delta$ function, as well as the sharp peak, leads to an oscillation in $S(k)$. In this way, it can be shown that the $\delta$ function at the bond-length causes oscillations of

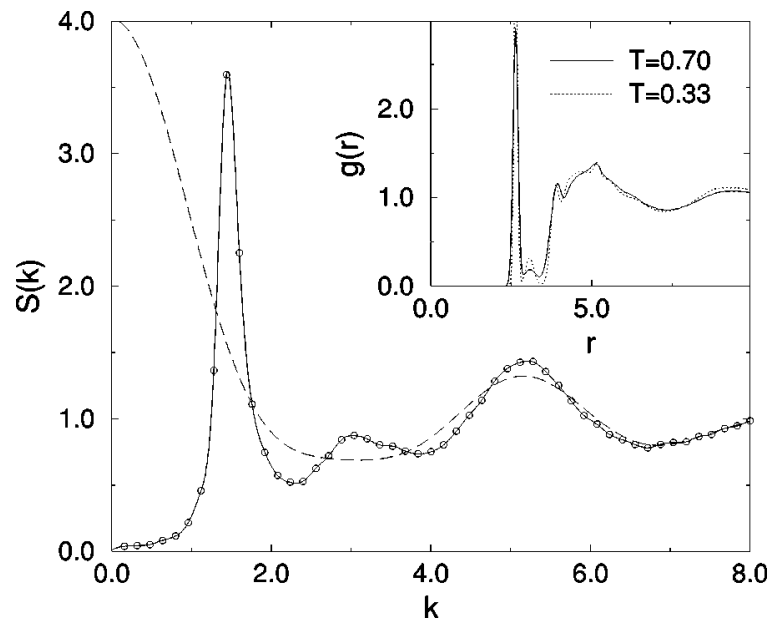

FIG. 2. Static structure factor $S(k)$ at $T=0.70$. The dashed line is the contribution to $S(k)$ from the first two peaks in $g(r)$. Inset: Radial distribution function $g(r)$ at temperatures $T=0.70$ and $T$ $=0.330$

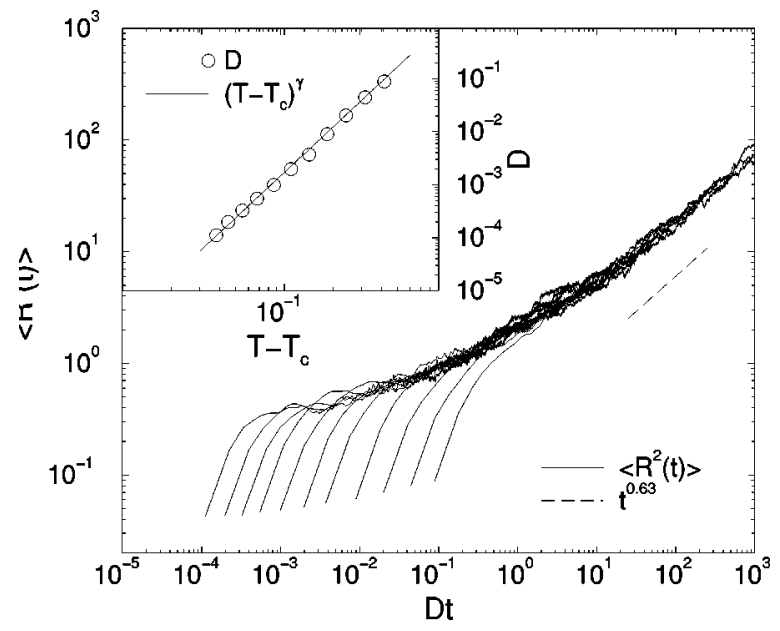

FIG. 3. Mean square displacement as a function of $D_{\alpha} t$ for each temperature measured between $T=0.70$ and $T=0.323$. Inset: Temperature dependence of the diffusion constant $D_{\alpha}$ defined in Eq. (17). The straight line is a fit according to Eq. (12) with $T_{c}$ $=0.285$ and $\gamma=2.85$.

which the first maximum appears at approximately $k$ $=5.2 \AA^{-1}$. The second peak of this oscillation falls out of the $k$ region measured in $S(k)$. The first peak of the oscillations originating from the sharp peak at $r=2.6 \AA$ is situated at approximately $k=3.1 \AA^{-1}$. The total contribution to the static structure factor of these two peaks is shown in Fig. 2. This contribution coincides with the static structure factor after approximately $k=5 \AA^{-1}$. From the difference between the solid line and the dashed line in Fig. 2, it is clear that the first two peaks in $S(k)$ are caused by intermolecular correlations.

\section{B. Mean square displacement}

A common way to analyze the single particle dynamics in real space is by calculating the mean square displacement. In polymers one can distinguish the displacement of the center of mass and the displacement of a single site. Since we are interested in self motion of the chain segments, we focus on the latter. The results of these calculations are shown in Fig. 3 for all the temperatures simulated, from $T=0.7$ (right) to $T=0.323$ (left). Here, the mean square displacement is plotted as a function of $D_{\alpha} t$, where $D_{\alpha}$ is a "diffusion" constant defined by

$$
\left\langle\Delta R(t)^{2}\right\rangle=\left(D_{\alpha} t\right)^{\alpha},
$$

in the long time limit. In this way the curves coincide in the Rouse-regime. Note that in this regime there is no normal diffusion, i.e., $\alpha \neq 1$. In unentangled polymer melts, $\alpha$ is typically about 0.6 , somewhat higher than the 0.5 of ideal Rouse dynamics [12]. In our simulations we found a value of 0.63 , which is the same as in an earlier study of poly(butadiene) [15] and in the bead-spring model [16], indicating that this value is not sensitive to the energy barriers for torsional rotation. On a longer timescale the displacement of the center of mass becomes relatively large compared with the displacement of a single site with respect to the center of mass. In that limit, the mean square displacement of a single site changes to a linear dependence on time. In our simulations 
no such crossover was found. This implies that the dynamics of the polymers is in the Rouse-regime during the entire simulation time. As a result, the diffusion constant $D_{\alpha}$ obtained using Eq. (17) is a diffusion constant for the Rouseregime, at all temperatures measured. When a crossover to a linear time dependence was observed for the mean square displacement, Eq. (17) could not be used simply in the long time limit, but should be used in the Rouse-regime.

In the inset of Fig. 3 the "diffusion" constant $D_{\alpha}$ is shown as a function of the temperature. According to MCT, the diffusion constant shows a power law behavior and becomes zero at a critical temperature $T_{c}$, Eq. (12). The straight line shows that this power law behavior holds for about three orders of magnitude. The parameter $t_{0}$ is assumed to be temperature independent. The parameters $T_{c}$ and $\gamma$ obtained in this way are 0.285 , corresponding to 215 $\mathrm{K}$, and 2.85 , respectively. This $\gamma$ is somewhat lower than the 3.2 found in the mean square displacement of poly(butadiene) [15], but higher than the 2.3 and 2.1 found by Bennemann et al. [16] in an NVT and NPT ensemble respectively. The latter are obtained in the real diffusive regime (i.e., $\alpha$ $=1$ ), but as we will see in the analysis of $F(k, t)$, where similar values are found, this cannot explain the difference. This shows the influence of intramolecular interaction on the slowing down near the glass transition. Although only three different polymers are compared, its seems that $\gamma$ is increasing as the polymer becomes stiffer. Note that poly(butadiene) has a very stiff double bond. In simple liquids, $D_{\alpha}$ is a real diffusion constant (i.e., $\alpha=1$ ) and it is shown in simulations that close to the critical temperature $T_{c}$, the temperature dependence of $D_{\alpha}$ is a power law. It appears that this result can be generalized to polymer dynamics, were the self-motion is determined by Rouse-like dynamics (i.e., $\alpha \neq 1$ ), but a power law behavior of $D_{\alpha}$ is still observed.

\section{Intermediate scattering function}

To get more detailed information about the self-motion of the chain segments in the undercooled regime, we calculated the self part of the intermediate scattering function, $F^{s}(k, t)$, using Eq. (1). In the remaining part of this article the superscript $s$ is dropped. $F(k, t)$ is calculated in a range from $k$ $=0.16 \AA^{-1}$, which is the smallest wave number accessible in our simulation, to $k=3.84 \AA^{-1}$, which is about three times the wave number of the first peak in $S(k)$. In Fig. 4 we show a typical result, $k=1.44 \AA^{-1}$, for all temperatures measured. The wave vector $k=1.44 \AA^{-1}$ corresponds to the first peak in the static structure factor. At low temperatures, $F(k, t)$ shows the typical features of glass forming liquids: A fast process, followed by a plateau, the $\beta$-relaxation, and finally the $\alpha$-relaxation. In simple monoatomic liquids, the $\alpha$-relaxation is related to the diffusive motion of the atoms and the $\beta$-relaxation is related to the movement of the atoms in their cage. In the case of polymers, one would expect that the $\alpha$-relaxation is related to the Rouse-like motions of the polymers, at least at small $k$ values.

To perform a detailed test of the MCT we start by analyzing the $\alpha$-relaxation. In this regime, the relaxation times are obtained by using a stretched exponent as a fit function. These relaxation times are obtained at different temperatures to determine the parameter $\gamma$. By using Eq. (7), we can

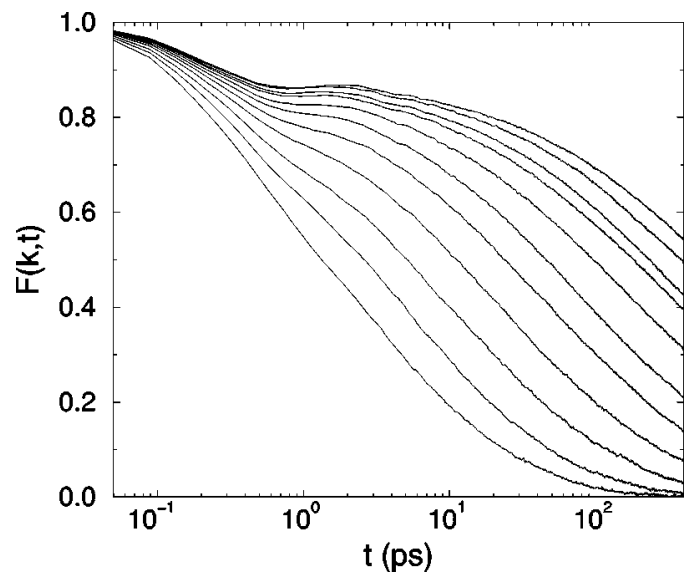

FIG. 4. Incoherent intermediate scattering function $F(k, t)$ at $k$ $=1.44 \AA^{-1}$, for each temperature measured between $T=0.70$ and $T=0.323$.

extract the values of the corresponding parameters $a$ and $b$, to describe the $\beta$-relaxation regime. In the remaining part of this paper the details of this procedure are described.

\section{1. $\alpha$-relaxation}

In the $\alpha$-relaxation regime, the second scaling law is one of the major results of the MCT. This scaling law, also referred to as the time-temperature superposition principle, has been shown to be valid for numerous types of liquids $[7,8,15,16]$, when the temperature is close to $T_{c}$. We illustrate this principle in Fig. 5 for $k=0.48,1.44$, and $2.88 \AA^{-1}$, where $F(k, t)$ is plotted as a function of $t / \tau$. For small $k$, this superposition principle holds for the entire temperature region measured, but on increasing $k$, this temperature region becomes smaller. For $k=1.44 \AA^{-1}$ and $k=2.88 \AA^{-1}$, the temperatures $T=0.7$ and $T=0.6$ do not obey this superposition principle and are not shown. For these temperatures the parameter $\beta$, Eq. (11), is larger. This indicates that, for $k$ $=1.44 \AA^{-1}$ and $k=2.88 \AA^{-1}$, these two temperatures fall out of the temperature region described by MCT.

The parameters $c_{k}, \tau_{k}$, and $\beta_{k}$ of the master function, Eq. (11), can be determined in the total $k$-range. We followed the procedure that the fit of this stretched exponent must be correct for $t \gg \tau$ and found that the length of the region of $F(k, t)$ that can be described with a stretched exponent is $k$ dependent. The largest regions were observed for large wave numbers, as shown in Fig. 5. The values of $c_{k}$ and $\beta_{k}$ obtained in this way are shown in Fig. 6. For $\mathrm{k} \rightarrow 0, \beta$ is getting close to 0.63 , which is the same value as has been found for $\alpha$ in the mean square displacement. This can be understood by using the Gaussian approximation, which becomes valid in the small $k$ region,

$$
F(k, t)=e^{-(1 / 6) k^{2}\left\langle\Delta R^{2}(t)\right\rangle}=e^{-(1 / 6) k^{2}\left(D_{\alpha} t\right)^{\alpha}} .
$$

Comparing the Gaussian approximation with a stretched exponent, it is easy to see that $\beta \rightarrow \alpha(=0.63)$ for small $k$ values. Hence, at small $k$ values, this stretched exponential behavior is simply a result of Rouse-like dynamics, and not caused by the glass transition. In the case of normal diffusion, $\alpha=1, \beta \rightarrow 1$ for small $k$ values [7]. On increasing $k, \beta$ is decreasing and becomes constant, $\beta \approx 0.41$, beyond $k$ 

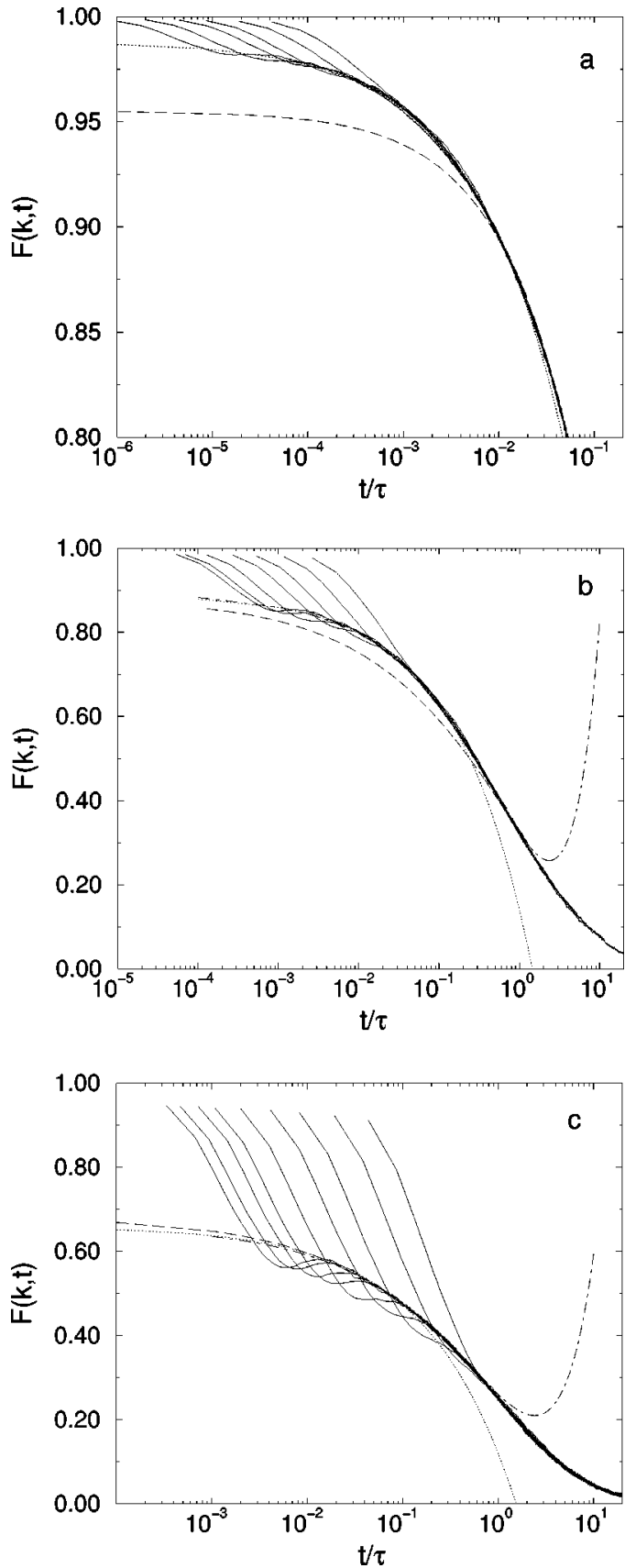

FIG. 5. Incoherent intermediate scattering function $F(k, t)$ as a function of $t / \tau$ for $k=0.48 \AA^{-1}$ (a), $k=1.44 \AA^{-1}$ (b), and $k$ $=2.88 \AA^{-1}$ (c). The dashed line is a fit according to a stretched exponent, Eq. (11), the dotted line is the Von Schweidler relaxation, Eq. (19), and the dotted-dashed line is a fit according to the Von Schweidler relaxation including the $t^{2 b}$ term.

$=1.5 \AA^{-1}$. In this region these values of $\beta$ are different from those obtained by Bennemann et al. [18] who observed values of about 0.75 at wave vectors corresponding to the first peak of $S(k)$, which are similar to $\beta$ 's obtained in simple liquids. Values of $\beta$ of about 0.40 are in good agreement with results of neutron scattering experiments [30], where this parameter ranges from 0.40 to 0.55 , depending on the type of polymer.

Another consequence of this approximation is that $\tau_{k}$ $\sim k^{-2 / 0.63}$ for $k \rightarrow 0$. In Fig. 7 it is shown that this approximation is correct until $k \approx 1 \AA^{-1}$ and that this region is in-

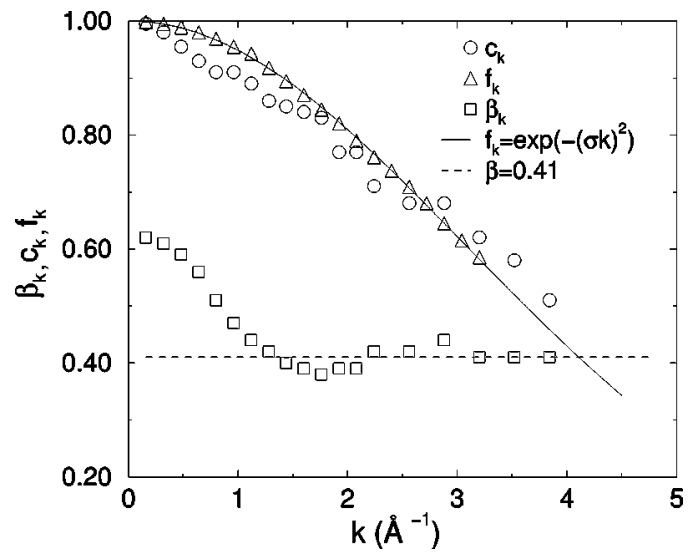

FIG. 6. $c_{k}, f_{k}$, and $\beta_{k}$ of Eqs. (11) and (19). The solid line is a Gaussian fit using $\sigma=0.23 \AA$ and the dashed line corresponds to $\beta=0.41$.

dependent of the temperature. These observations show that it can be misleading to use values of $\beta$ obtained near the first peak of $S(k)$ in the Gaussian approximation. In this way $\beta$ is underestimated, in this case 0.40 instead of 0.63 , and therefore the $k$ dependence of $\tau$ is overestimated. This could probably explain the small deviations from the Gaussian approximation found by Arbe et al. [30] in their study of the nature of the $\alpha$-relaxation (homogeneous or heterogeneous). After $k \approx 1 \AA^{-1}$ there is a somewhat stronger $k$ dependence. This differs substantially from the observations of Bennemann et al. [18], who found a crossover to the large $k$ limit of MCT; $\tau \sim k^{-b}$, even before the first peak in $S(k)$. Such a crossover is not observed in our simulations and in experiments on glass forming polymers [30]. This, together with the different $k$ dependence of $\beta$ shows the influence of a more realistic model on the dynamics of glass forming polymers, and demonstrates that a simple model is not sufficient in this $k$ region. The values of $c_{k}$ [cf. Eq. (11)] show roughly a Gaussian $k$ dependence.

When $\beta_{k}$ and $c_{k}$ are known, the general form of the stretched exponent is known and $\tau$ can be obtained at each temperature. In Fig. 8 this temperature dependence is shown for $k=0.48,1.44$, and $2.88 \AA^{-1}$. The straight lines corre-

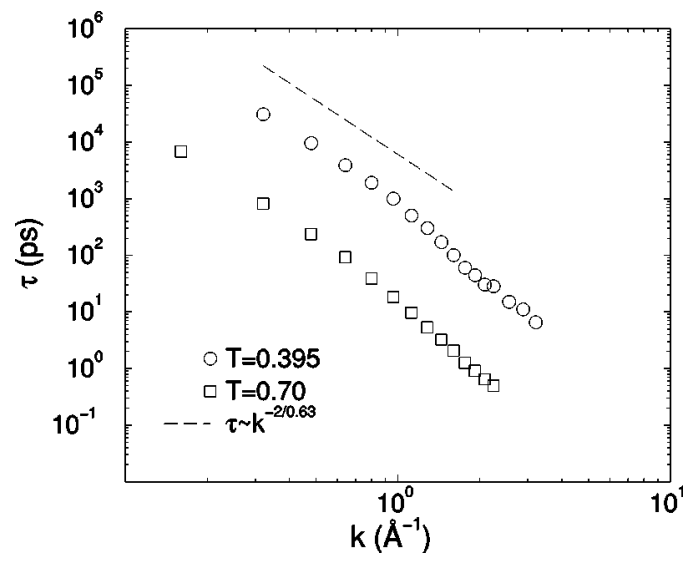

FIG. 7. $\tau_{k}$ as a function of $k$ for $T=0.330$ and $T=0.70$. The dashed line corresponds to the $k$ dependence of the Gaussian Approximation, using $\beta=0.63$ as found in the mean square displacement. 


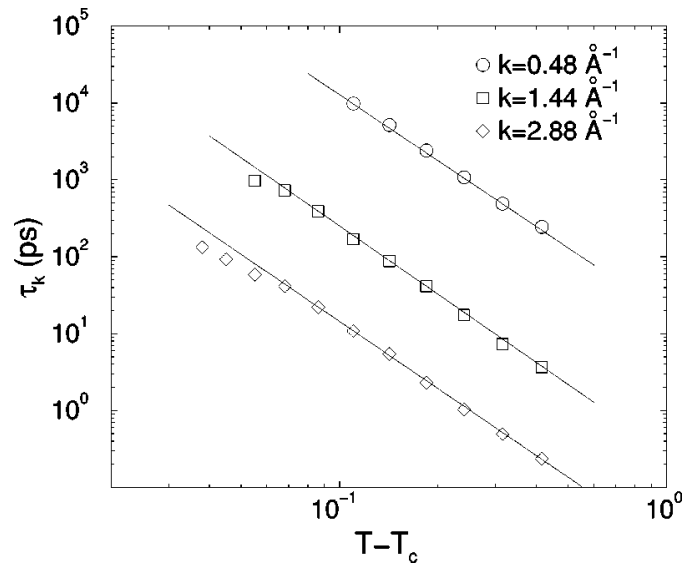

FIG. 8. Temperature dependence of the relaxation times $\tau_{k}$ for $k=0.48,1.44$, and $2.88 \AA^{-1}$. The straight lines are fits according to Eq. (12) using $T_{c}=0.28$ and $\gamma=2.85\left(k=0.48 \AA^{-1}\right), \gamma$ $=2.95\left(k=1.44 \AA^{-1}\right)$, and $\gamma=2.90\left(k=2.88 \AA^{-1}\right)$.

spond to a power law behavior. As in the case of the mean square displacement, we used $T_{c}=0.285$, corresponding to $215 \mathrm{~K}$. Using Eq. (12), and again taking $t_{0}$ temperature independent, $\gamma$ can be obtained. We found 2.85 for $k$ $=0.48 \AA^{-1}, 2.95$ for $k=1.44 \AA^{-1}$, and 2.90 for $k$ $=2.88 \AA^{-1}$. These $\gamma$ 's are approximately the same as $\gamma_{D}$. This differs from the observations of Bennemann et al. [18], who found a difference between the temperature dependence of the diffusion constant $D_{\alpha}$ and the $\alpha$-relaxation. However, at the same time they show that the Gaussian approximation is valid for small wave vectors, from which one can conclude that $\gamma_{D}$ and $\gamma$ should be the same, as observed in our simulations. We now neglect this small $k$ dependence in $\gamma$ and use $\gamma=2.90$, which is just the average of these three $k$ values. The values of $\gamma$ obtained from $k=0.48 \AA^{-1}$ and $k$ $=1.44 \AA^{-1}$ differ by less than $2 \%$ from this average.

\section{2. $\beta$-relaxation}

Once $\gamma$ is known, one can obtain $a, b$, and $\lambda$ by using Eqs. (7) and (13). This leads to four parameters which, according to MCT, describe the $\beta$-relaxation regime. These are

$$
\begin{aligned}
& \gamma=2.90, \\
& \lambda=0.81, \\
& a=0.27, \\
& b=0.46 .
\end{aligned}
$$

As mentioned before, a good way to show the validity of the MCT is to use these values in the expansions of the $\beta$-correlator, Eqs. (5) and (6). We start with the long time part of the $\beta$-relaxation. By rewriting Eq. (6) and using $B_{1}$ $=0$, we obtain the Von Schweidler relaxation

$$
F(k, t)=f_{k}-h_{k}(t / \tau)^{b}
$$

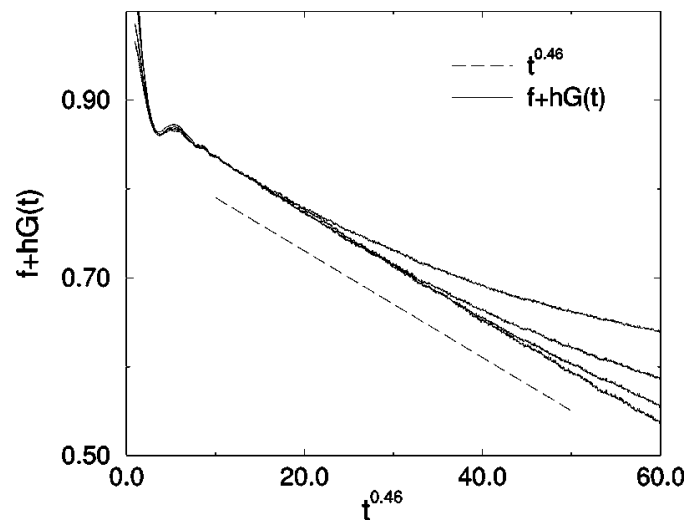

FIG. 9. $f+h G(t)$ as a function of $t^{0.46}$, obtained from $F(k, t)$ for $k=2.72,2.08,1.44$, and $0.80 \AA^{-1}$ (top to bottom) at temperature $T=0.323$. The dashed line corresponds to the Von Schweidler relaxation $F(k, t)=f_{k}-h_{k} t^{b}$, using $b=0.46$.

This relaxation is also shown in Fig. 5 for all three $k$ values. For $k=0.48 \AA^{-1}$ and $k=1.44 \AA^{-1}$, this Von Schweidler relaxation gives a perfect fit for the long-time part of the $\beta$-relaxation for more than two decades in time at the lowest temperatures. For $k=0.48 \AA^{-1}$ this region extends over more then three decades in time. For $k=2.88 \AA^{-1}$, the Von Schweidler relaxation is only valid in a very short time regime. Here, the long-time part of the $\beta$-relaxation can still be described accurately by a stretched exponent. In this $k$ range, the $\beta$-relaxation regime is probably to small to observe the Von Schweidler relaxation. When we look at small $k$ values, where the Von Schweidler relaxation is observed, it is clear that the time regime were it is valid becomes smaller as the temperature is increased. Therefore, it is very likely that closer to $T_{c}$, the extend of the Von Schweidler relaxation becomes larger, even at larger $k$ values. However, it is also possible that MCT is not able to describe this $k$ region, because of the importance of intramolecular correlations. But one has to keep in mind that the second scaling law and the power law behavior of the relaxation time are still obeyed at larger $k$ values. Using Eq. (19), we determined $f_{k}$ in the entire $k$ region measured and the results are shown in Fig. 6. Unlike $c_{k}, f_{k}$ coincides perfectly with the Gaussian curve.

The fact that the $\beta$-relaxation regime can be described by MCT was also observed by Eliasson et al., who measured the dielectric susceptilibity of amorphous poly(ethylene terephthalate) (PET) [31]. They found that above $T_{c}$, the $\beta$-relaxation could be described with $\lambda=0.82$, corresponding to $\gamma=3.0$ and $b=0.44$. These values are close to the ones we found in analyzing $F(k, t)$. The exponent parameter $\lambda$ found in our analysis is quite different from the one obtained by Bennemann et al. [18], who obtained $\lambda=0.635$ corresponding to $\gamma=2.07$ and $b=0.75$. Again, this indicates the influence of the intramolecular forces on the dynamics close to the glass transition.

To get more information about the long-time part of the $\beta$-relaxation, $F(k, t)$ is plotted in Fig. 9 for different $k$ values, as a function of $t^{b}$, at the lowest temperature measured. $F(k, t)$ is rescaled to fall on $F(k, t)$ for $k=1.44 \AA^{-1}$. So in fact, we plotted $f+h G(t)$ obtained from different $k$ values, where $f$ and $h$ are equal to $f_{k}$ and $h_{k}$ for $k=1.44 \AA^{-1}$. For $k=0.90 \AA^{-1}$, the lowest curve, $F(k, t)$ becomes a straight 


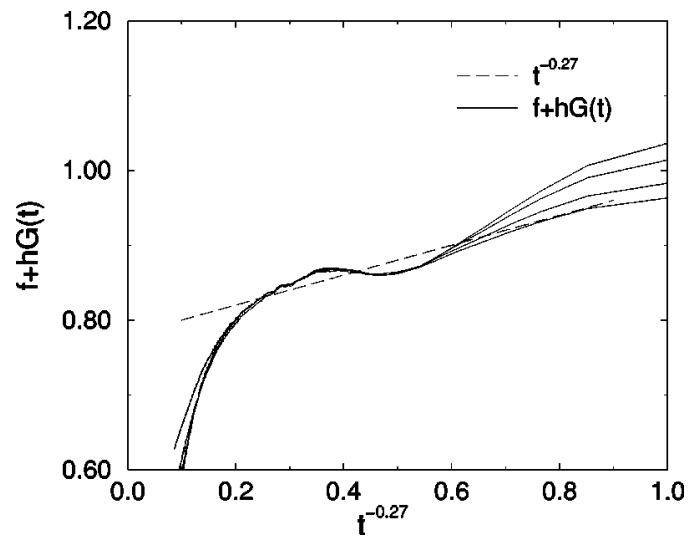

FIG. 10. $f+h G(t)$ as a function of $t^{-0.27}$, obtained from $F(k, t)$ for $k=2.72,2.08,1.44$, and $0.80 \AA^{-1}$ (top to bottom) at temperature $T=0.323$. The dashed line corresponds to the critical decay $F(k, t)=f_{k}+h_{k} t^{-a}$, using $a=0.27$.

line indicating the Von Schweidler relaxation over a large time region. On increasing $k$, this region becomes smaller. The point where the different $G(t)$ 's leave the straight line is where the $\alpha$-relaxation sets in. Since the relaxation times of the $\alpha$-relaxation are $k$ dependent, this point is also $k$-dependent. The length of the Von Schweidler relaxation can be increased using a $k$ dependent $b$, but this is in contradiction with the predictions of MCT, which states that $b$, as well as $a, \lambda$, and $\gamma$ are $k$ independent. In other simulations a $k$ dependent $b$ is found [7] and, for obvious reasons, this is called the effective Von Schweidler parameter $b^{\prime}$. A way to avoid $k$ dependent parameters is to add a term proportional to $t^{2 b}$ to Eq. (19). This term is found analytically by Franosch et al. [32] as a first order correction to MCT, further away from the critical temperature. For $k=1.44 \AA^{-1}$ and $k$ $=2.88 \AA^{-1}$ the results of these fits are also shown in Fig. 5 . It is clearly visible that including the $t^{2 b}$ term extends the validity of the fit over almost one decade. Also, the parameters $B_{1}$ increase by about $10 \%$ for both wave vectors. However, no significant changes were observed in $f_{k}$.

The first part of the $\beta$-relaxation regime can be analyzed in a similar way. By rewriting Eq. (5) and using $A=0$, we obtain the critical decay

$$
F(k, t)=f_{k}+h_{k}\left(t_{0} / t\right)^{a} .
$$

So, we rescale $F(k, t)$ for different $k$ values, as in the analysis of the long-lime part of the $\beta$-relaxation, and plot these as a function of $t^{-a}$. This is shown in Fig. 10. In the time region of the critical decay we should expect a straight line, similar as in the case of the Von Schweidler relaxation. It is clearly visible that no such area exists, even when we try different values for $a$. The reason for this are the oscillations around $t=1 \mathrm{ps}$, also present in Fig. 5 and Fig. 9. These oscillations are believed to be related to the boson peak [33], found in many other glasses and glass formers, in both simulations and experiments. A detailed study of the boson peak falls outside the scope of this article.

\section{SUMMARY AND CONCLUSION}

We performed a detailed analysis of the dynamical properties of glass-forming polymers using molecular dynamics. The main goal was to determine to what extent the MCT, which is derived for monoatomic liquids, is able to describe the dynamics of realistic polymer melts in the undercooled regime. In this paper we focused on the self-motion of the individual sites in the polymers using the mean square displacement and the incoherent intermediate scattering function. The latter is calculated in a large $k$ range.

By analyzing the mean square displacement we find Rouse-like dynamics in the entire time scale of our simulations. The corresponding "diffusion" constant follows a power law behavior. It is shown that in the $\alpha$-relaxation regime the second scaling law of the MCT is valid in the entire $k$ range. The relaxation times follow the same power law behavior as the diffusion constant $D_{\alpha}$, i.e., $\gamma_{D}$ and $\gamma$ are the same. Once $\gamma$ is known, $a$ and $b$ are determined and checked using the Von Schweidler relaxation and the critical decay. Using $b$, the last part of the $\beta$-relaxation is described accurately, but no critical decay is observed. Finally, factorization of $k$ and $t$ in the $\beta$-relaxation, Eq. (2), is found.

These results show that the MCT can be used to describe the dynamics of realistic polymer melts in the undercooled regime. The only way the typical Rouse-like motion of the polymers can be observed is in the mean square displacement of the sites in the polymer chain and in the small $k$ limit of $F(k, t)$. Here $\beta$ reaches the value of 0.63 as predicted from the mean square displacement. Also in this limit, where the $\alpha$-relaxation is caused by a Rouse-like motion, MCT holds. The last part of the $\beta$-relaxation, which describes the escape of a particle out of its cage is described well by MCT. This leads to the conclusion that the cage effect, leading to the structural arrest in the idealized MCT, is similar in polymer melts and monatomic liquids. It appears that the geometry of the polymer chain, which influences the short range order as seen in the radial distribution function, does not influence the cage-effect significantly. This is in agreement with simulations of diatomic molecules [8] and water [9], also having a different geometry, which show similar results. Schweizer [34,35] studied the short time dynamics of a model in which both Rouse dynamics and mode coupling terms were included. He found that the mean square displacement is proportional to $t^{9 / 32}$ at a short timescale. No such behavior was found in our simulations.

In addition to the mode-coupling analysis, we compared our results with a detailed MD-study of a glass forming polymers melt performed by Bennemann et al. [18], and to experimental results. We showed that the intramolecular interaction, which is the main difference between the two models, leads to a better agreement with experimental results on the following cases: (i) The stretching parameter $\beta$ is similar, around 0.4 , as found in neutron scattering experiments. (ii) The $k$ dependence of the relaxation times of the $\alpha$-relaxation agrees with experimental results, and (iii) the exponent parameter $\lambda$ obtained in this study $(0.81)$ is close to the experimentally obtained value, found in glass forming PET (0.82).

In the analysis described in this paper we made use of the relationship between the parameters $a, b, \gamma$, and $\lambda$. In principle, according to MCT, these parameters can be calcu- 
lated once the $S(k)$ is known. Also, the nonergodicity parameter $f_{k}$ can be calculated. The latter is calculated by Nauroth and Kob [36] in the case of a binary liquid. In this way, more information about the validity of the MCT in the case of polymers can be obtained. This, in addition to the analysis of the coherent part of the intermediate scattering function, will be the subject of future work.

In summary, we performed a detailed analysis of the dynamics of an undercooled polymer melt within the framework of MCT. The incoherent part of the intermediate scattering function is calculated and we show that MCT, which is derived to describe the dynamics of simple liquids, can be used to understand the dynamics of undercooled polymer melts. The coherent part of the intermediate scattering function, together with a more quantitative analysis, will be the subject of future work.

\section{ACKNOWLEDGMENTS}

This work is part of the research program of the Division for Chemical Sciences $(\mathrm{CW})$ and was made possible by financial support from the Netherlands Organization for Scientific Research (NWO). We thank the center for High Performance Applied Computing $(\mathrm{HP} \alpha \mathrm{C})$ for generous allocation of computing time on the CRAY-T3E. The authors would like to thank Dr. I.M. de Schepper for useful discussions.
[1] E. Leutheusser, Phys. Rev. A 29, 2765 (1984).

[2] U. Bengtzelius, W. Götze, and A. Sjölander, J. Phys. C 17, 5915 (1984).

[3] See W. Götze, in Liquids, Freezing and the Glass Transition, edited by J. P. Hansen, D. Levesque, and J. Zinn-Justin (NorthHolland, Amsterdam, 1991).

[4] J. Ullo and Sidney Yip, Phys. Rev. A 39, 5877 (1989).

[5] M.J.D. Brakkee and S.W. de Leeuw, J. Phys.: Condens. Matter 2, 4991 (1990).

[6] Walter Kob and Hans C. Andersen, Phys. Rev. Lett. 73, 1376 (1994).

[7] Walter Kob and Hans C. Andersen, Phys. Rev. E 52, 4134 (1995).

[8] Stefan Kämmerer, Walter Kob, and Rolf Schilling, Phys. Rev. E 58, 2131 (1998).

[9] F. Sciortino, P. Gallo, P. Tartaglia, and S.-H. Chen, Phys. Rev. E 54, 6331 (1996).

[10] A. Arbe, D. Richter, J. Colmenero, and B. Farago, Phys. Rev. E 54, 3853 (1996).

[11] A. Arbe, U. Buchenau, L. Willner, D. Richter, B. Farago, and J. Colmenero, Phys. Rev. Lett. 76, 1872 (1996).

[12] M. Doi and S.F. Edwards, The Theory of Polymer Dynamics (Clarendon Press, Oxford, 1986).

[13] Kurt Kremer and Gary S. Grest, J. Chem. Phys. 92, 5057 (1990).

[14] Ryong-Joon Roe, J. Chem. Phys. 100, 1610 (1994).

[15] A. van Zon and S.W. de Leeuw, Phys. Rev. E 58, R4100 (1998).

[16] Christoph Bennemann, Wolfgang Paul, Kurt Binder, and Burkhard Dünweg, Phys. Rev. E 57, 843 (1998).

[17] Jörg Baschnagel, Phys. Rev. B 49, 135 (1994).

[18] C. Bennemann, J. Baschnagel, and W. Paul, e-print cond-mat/9809335.
[19] W. Paul, G.D. Smith, D.Y. Yoon, B. Farago, S. Rathgeber, A. Zirkel, L. Willner, and D. Richter, Phys. Rev. Lett. 80, 2346 (1998).

[20] B. Mos, P. Verkerk, S. Pouget, A. van Zon, G.-J. Bel, S. W. de Leeuw, and C. D. Eisenbach (unpublished).

[21] J.P. Hansen and I.R. McDonald, Theory of Simple Liquids (Academic Press, New York, 1986).

[22] Umberto Balucani and Marco Zoppi, Dynamics of the Liquid State (Clarendon Press, Oxford, 1994)

[23] W. Götze, Z. Phys. B 60, 195 (1985).

[24] R. Zorn, A. Arbe, J. Colmenero, D. Richter, and U. Buchenau, Phys. Rev. E 52, 781 (1995).

[25] U. Bengtzelius, Phys. Rev. A 34, 5059 (1986).

[26] See R.-J. Roe, in Advances in Polymer Science 116, edited by L. Monnerie and U.W. Suter (Springer-Verlag, Berlin, 1994).

[27] Sylvie Neyertz and David Brown, J. Chem. Phys. 102, 9725 (1995).

[28] S.W. de Leeuw, J.W. Perram, and H.G. Petersen, J. Stat. Phys. 61, 1203 (1990).

[29] William G. Hoover, Phys. Rev. A 31, 1695 (1985).

[30] A. Arbe, J. Colmenero, M. Monkenbusch, and D. Richter, Phys. Rev. Lett. 81, 590 (1998).

[31] H. Eliasson, B.-E. Mellander, and L. Sjögren, J. Non-Cryst. Solids 235-237, 101 (1998).

[32] T. Franosch, M. Fuchs, W. Götze, M.R. Mayr, and P. Singh, Phys. Rev. E 55, 7153 (1997).

[33] See, e.g., Structure and Dynamics of Glasses and Glass Formers, edited by C.A. Angell, K.L. Ngai, J. Kieffer, T. Egami, and G.U. Nienhaus (Materials Research Society, Pittsburgh, 1997).

[34] Kenneth S. Schweizer, J. Chem. Phys. 91, 5802 (1989).

[35] Kenneth S. Schweizer, J. Chem. Phys. 91, 5822 (1989).

[36] Markus Nauroth and Walter Kob, Phys. Rev. E 55, 657 (1997). 